\section{Pacific Northwest}

National Laboratory

Operated by Battelle for the

U.S. Department of Energy

\title{
Demonstration of Smart Building Controls to Manage Building Peak Loads: Innovative Non-Wires Technologies
}

\author{
S. Katipamula \\ D. Hatley
}

November 2004

Prepared for the

U.S. Department of Energy

under Contract DE-AC05-76RL01830 


\title{
Demonstration of Smart Building Controls to Manage Building Peak Loads: Innovative Non-Wires Technologies
}

\author{
S. Katipamula \\ D. Hatley
}

November 2004

Prepared for

the U.S. Department of Energy

under Contract DE-AC05-76RL01830

Pacific Northwest National Laboratory

Richland, Washington 99352 


\section{Summary}

The Bonneville Power Administration (BPA) is exploring a variety of innovative non-wires technology alternatives that can help address transmission congestion in the Pacific Northwest. Distributed energy resources (DERs) are one such set of technologies that can help address imbalance between supply and demand. DERs along with smart building controls can provide temporary delay or in some case totally eliminate the need for construction of new transmission and distribution lines. DER technologies include distributed generation, storage, and demand responsive loads.

As a part of the non-wires solutions effort, BPA in partnership with Pacific Northwest National Laboratory (PNNL) is exploring the use of two DER technologies in the City of Richland. In addition to demonstrating the usefulness of the two DER technologies in providing peak demand relief, evaluation of remote direct load control (DLC) is also one of the primary objectives of this demonstration. The concept of DLC, which is used to change the energy use profile during peak hours of the day, is not new. Many utilities have had success in reducing demand at peak times to avoid building new generation. It is not the need for increased generation that is driving the use of direct load control in the Northwest, but the desire to avoid building additional transmission capacity. The peak times at issue total between 50 and 100 hours a year. A transmission solution to the problem would cost tens of millions of dollars ${ }^{\mathrm{a}}$. And since a "non wires" solution is just as effective and yet costs much less, the capital dollars for construction can be used elsewhere on the grid, where building new transmission is the only alternative. If by using DLC, the electricity use can be curtailed, shifted to lower use time periods or supplemented through local generation, the existing system can be made more reliable and cost effective.

\footnotetext{
${ }^{\mathrm{a}}$ Although it is difficult to quantify the cost of building new transmission lines, the quote from the BPA administrator provides some sense - "It is not unusual for construction of a transmission line to cost upwards of \$100 million or more. For example, we are nearing completion on our new 84-mile Grand Coulee-Bell line in Washington. Its price tag will be around $\$ 175$ million. It can be even more challenging to build in urban areas. For example, the 9-mile line for Kangley-Echo Lake cost nearly \$60 million due to extensive mitigation requirements."
} 


\section{Table of Contents}

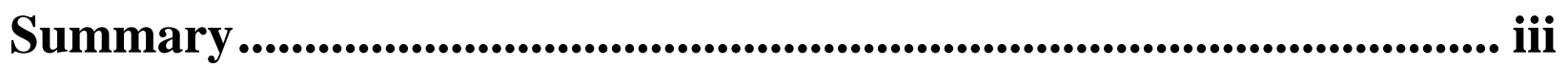

Microturbine Demonstration Project......................................................1

Remote Load Shedding Project ......................................................................2

Smart Building Monitoring and Control ...............................................3

Summary of Results .......................................................................................6 


\section{Figures}

Figure 1. Example Integration of Control Network from Different Building with Internet ........................ 3

Figure 3. Overview of the Remote Monitoring and Controls ............................................................. 4

Figure 3. Screen Shot of the Website Built for Remote Monitoring and Control...................................... 5

\section{Tables}

Table 1. Summary of Demand and Electricity Use Reduction from Direct Load Control ......................... 6 


\section{Microturbine Demonstration Project}

The first project explores the use of a distributed generation asset during peak hours. The goal of this project is to demonstrate a remote access system that controls and monitors a distributed generation (DG) system delivering grid-connect electrical power from a commercial building site. Furthermore, it seeks to evaluate whether and to what extent this approach is a reliable and quantifiable non-wires resource.

To evaluate this approach, a 30-kW Capstone microturbine system was installed at a commercial building site in Richland. The microturbine controls were integrated with PNNL's existing enterprise-wide energy management and controls system (EMCS) - $\underline{\text { Johnson Controls Inc. Metasys }}{ }^{\circledR}$ (JCI). In addition, PNNL also developed a web interface that provides easy access to the enterprise EMCS. This integration provides BPA with the ability to monitor and start/stop the turbine remotely. Details of the integration are provided later in this report. The microturbine has been in operation 15 to 20 hours a week since mid July 2004 providing on average about $27 \mathrm{~kW}$ of load relief. 


\section{Remote Load Shedding Project}

The second project concerns demonstrating and quantifying the peak electricity-use reduction that can be achieved in a single building. In addition, BPA also wanted to demonstrate a smart building control system that provides both control and monitoring of dispersed commercial building electric loads through the use of Internet addressable devices, and evaluate whether and to what extent this approach is a reliable and quantifiable resource for the non-wires solutions effort. In partnership with BPA, PNNL developed and implemented load control algorithms on four buildings on the PNNL campus. Like the microturbine project, BPA was provided with the ability to monitor and trigger the load controls remotely. This was possible because the enterprise-wide EMCS was connected to the Internet.

Four buildings from the PNNL campus were selected for the load shedding project. The loads controlled include: 1) rooftop units (10 kW), 2) duty cycling of air handling units (20 kW), 3) cooling pond pumps $(100 \mathrm{~kW})$ and 4$)$ chiller and air handler controls (130 kW). The controllers in each building were preprogrammed to perform a sequence of operations when the load control was initiated, including remaining offline for a 30-minute period. During the 30-minute curtailment period, if the indoor temperatures (or pond water temperature, in case of item 3 above) rise to a preset upper limit, the equipment turns ON automatically.

Much of the load shedding events were in the afternoon, which coincided with the transmission and distribution peaks of BPA. Extending the target load shedding time periods for 1, 2 and 3 hour durations year-round will be explored in 2005, recognizing the time of day that events occur, and impacts on systems and tenants. 


\section{Smart Building Monitoring and Control}

To be able to remotely monitor and control building systems, the operator needs access to the building control network. Access can be through the Internet by connecting the control network to the Internet or direct access to the EMCS through other means (for example, over a telephone line using a modem). In the recent years, the standard practice for remote monitoring and control of building systems (including distributed generation assets) over the Internet has been through the use of a hardware gateway or a protocol converter that integrates the existing building control network with an Intranet or the Internet, as shown in Figure 1. This approach, however, is expensive because a gateway is needed at each building. In addition, the compatibility of gateways with different versions of EMCS software is not always what the manufactures claim it to be, resulting in problems that are not discovered until the gateway is installed.

The schematic of the approach used for the demonstration project is shown in Figure 2. Note that the approach does not depend on the use of gateways or protocol converters at each building; the integration of the control network with the Intranet is accomplished by using the native JCI network controllers, which are part of the basic enterprise control operations.

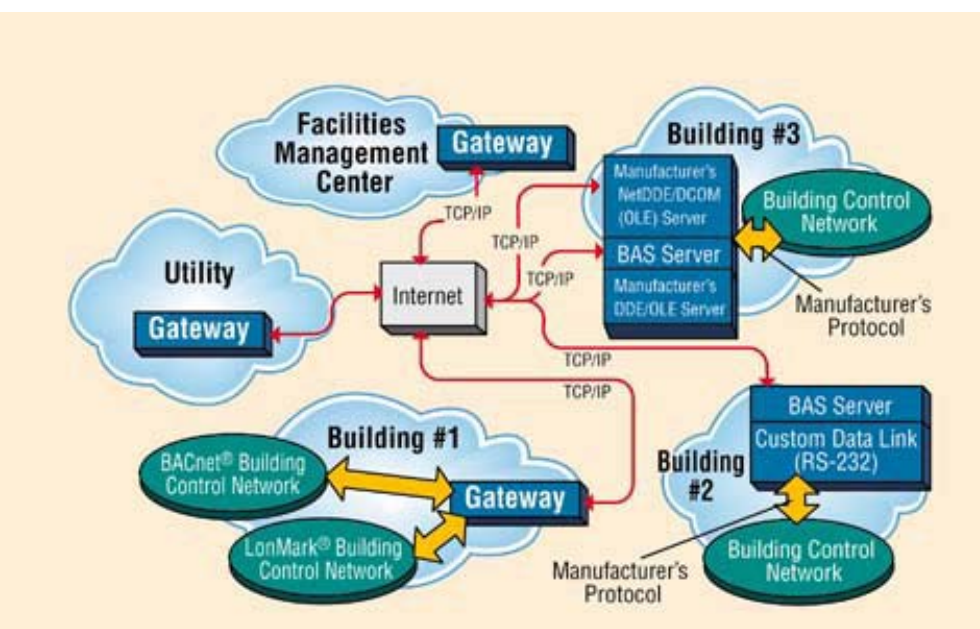

Figure 1. Example Integration of Control Network from Different Building with Internet

For the demonstration project, a web-server is hosted on a workstation, which also had a Microsoft $\mathrm{SQL}^{\mathrm{TM}}$ server $^{\mathrm{b}}$. In addition to storing the start/stop times of the curtailment events, the database also stores other historical data (ambient temperatures, electricity consumption). All communications between the web server and building controllers is through the OPC (OLE for Process Controls) ${ }^{\mathrm{c}}$ communication standard. The OPC standard is based on client/server architecture. Johnson Controls provides the OPC

\footnotetext{
${ }^{\mathrm{b}}$ Any ODBC (Open DataBase Connectivity) compliant database could have been used for the project.

${ }^{\mathrm{c}}$ Definition quoted from www.opc.org website - "Originally based on Microsoft's OLE (Object Link and Embedding) COM (component object model) and DCOM (distributed component object model) technologies, the specification defined a standard set of objects, interfaces and methods for use in process control and manufacturing automation applications to facilitate interoperability. The COM/DCOM technologies provided the framework for software products to be developed.”
} 


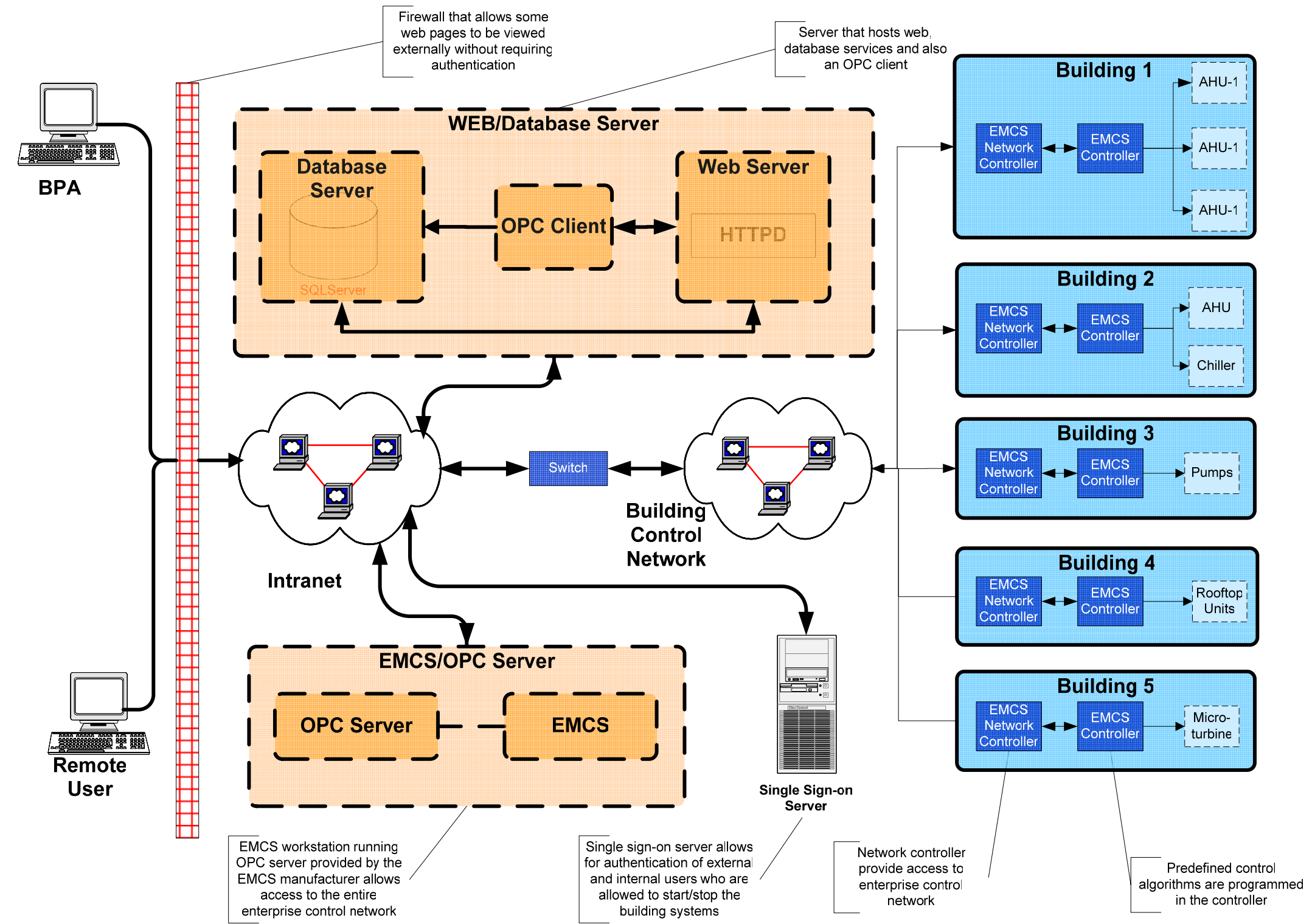

Figure 2. Overview of the Remote Monitoring and Controls 
server software that is typically run on an operator's workstation, as shown in Figure 2. Although an OPC client can be developed in house, for this project a commercial OPC client is used. When a request for data is made by the web-server, the request goes to the OPC client, which in turn passes the request to the OPC server running on an operator workstation. The OPC server then retrieves data from (or passes the control signal to) the relevant controller or sensor on the control network and then passes the information to the web server through the OPC client.

The monitoring portion of the website is open to the general public. Although the web-server is inside the PNNL firewall, the public can access the website (http://bpanws.pnl.gov) from outside the firewall through a reverse-proxy server (a screen shot of the website is shown in Figure 3). However, the controls portion of the website requires authentication for access, which is handled by PNNL's single sign-on server. The single sign-on server authenticates that the user has permission to start/stop the system. This type of authentication may not generally be available at most commercial buildings, but there are other forms of secure authentication systems that serve the same purpose.

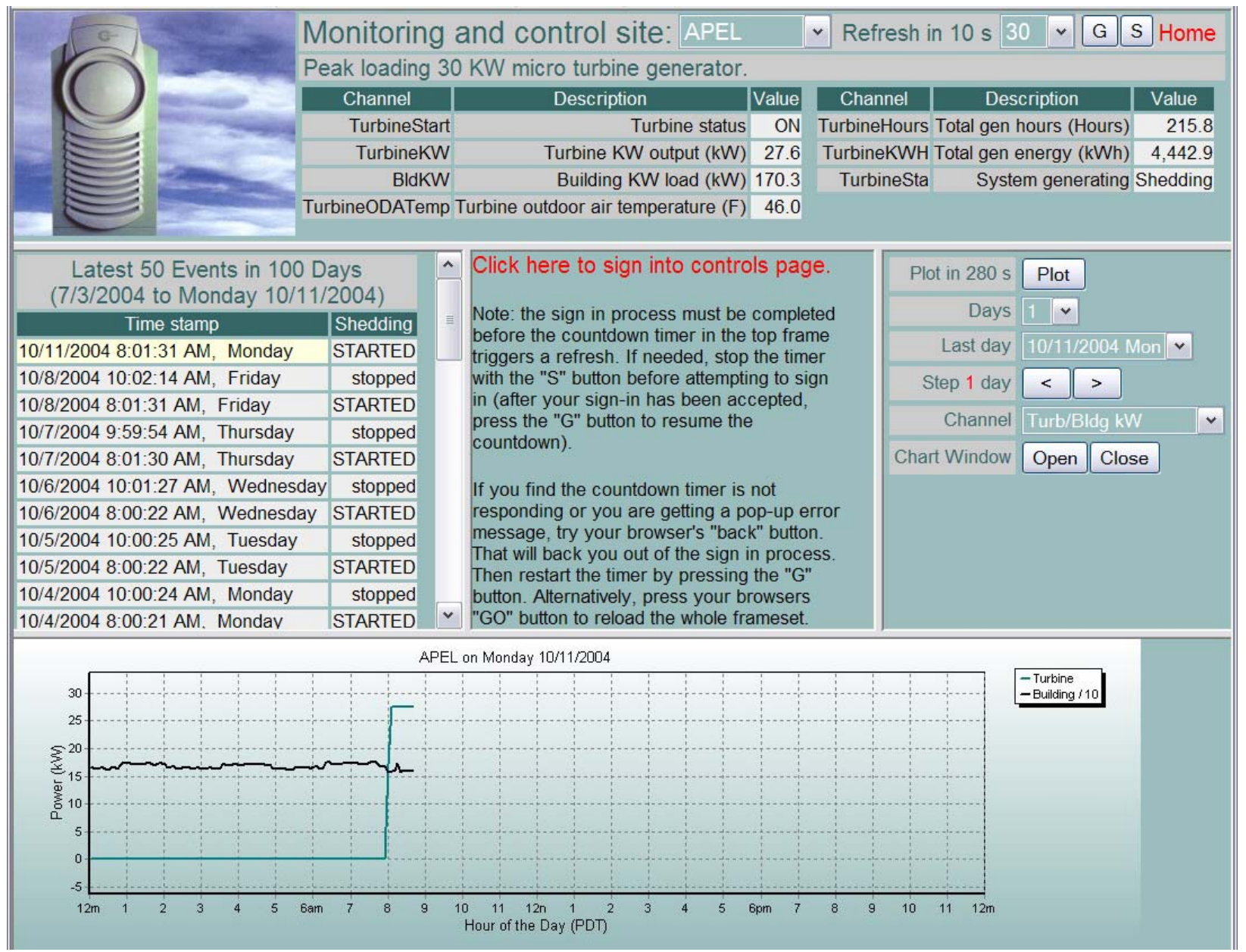

Figure 3. Screen Shot of the Website Built for Remote Monitoring and Control 


\section{Summary of Results}

Preliminary results from the two projects are summarized Table 1 below. On average, the demand reduction on the BPA transmission grid as result of the use of non-wires solutions is between $175 \mathrm{~kW}$ and $275 \mathrm{~kW}$. Although we have not quantified the effects on indoor comfort at this point, the building occupants and the building operators have not reported any comfort problems. We will quantify the change in comfort by monitoring the room temperature in 2005.

Table 1. Summary of Demand and Electricity Use Reduction from Direct Load Control

\begin{tabular}{|c|c|c|c|}
\hline Building & Type of Load & $\begin{array}{l}\text { Typical Demand } \\
\text { Reduction }(\mathbf{k W})\end{array}$ & $\begin{array}{l}\text { Typical Electricity- } \\
\text { Use Reduction }^{1} \\
\text { (kWh) }\end{array}$ \\
\hline Building 1 & Rooftop units & 10 & 5 \\
\hline Building 2 & $\begin{array}{l}\text { Duty cycling of air handling } \\
\text { units (AHUs) }\end{array}$ & 20 & 10 \\
\hline Building 3 & Cycling cooling pond pumps & 100 & 50 \\
\hline Building 4 & Chiller and AHU & 30 to 130 & 15 to 65 \\
\hline Building 5 & Microturbine & $27 \mathrm{~kW}$ & 108 \\
\hline Total & & 187 to 287 & 188 to 238 \\
\hline
\end{tabular}

${ }^{1}$ The load shedding event is pre-programmed to be 30 minutes in duration and the microturbine generation is pre-programmed to be about 4 hours long. 